\title{
碱炭比及活化温度对稻壳活性炭极微孔的影响
}

\author{
李大伟 ${ }^{1,2}$, 马腾飞 ${ }^{1,3}$, 田原宇 ${ }^{1,2}$, 朱锡锋 ${ }^{4}$, 乔英云 ${ }^{2}$
}

(1. 中国石油大学(华东) 化工学院, 青岛 266580; 2. 山东科技大学 低碳能源化工重点实验室, 青岛 266590; 3. 中 国石油天然气股份有限公司辽阳石化分公司, 辽阳 111003; 4. 中国科技大学 安徽省生物质洁净能源重点实验室, 合肥 230026)

摘 要: 采用 $\mathrm{N}_{2}$ 吸附、 $\mathrm{CO}_{2}$ 吸附和热重红外联用等技术手段, 考察了在 $\mathrm{KOH}$ 活化稻壳炭的过程中碱炭比和活化温 度对活性炭极微孔的影响。结果表明: 在不同碱炭比 $(0.6: 1 \sim 3: 1)$ 和活化温度 $\left(640 \sim 780^{\circ} \mathrm{C}\right)$ 下制备的稻壳活性炭, 极 微孔主要分布在 $0.42 \sim 0.70 \mathrm{~nm}$ 。当碱炭比增加时, 极微孔孔容先增大后减小; 而当活化温度升高时, 极微孔孔容呈 降低趋势。极微孔率随碱炭比或活化温度的升高而单调递减。在活化温度为 $640^{\circ} \mathrm{C}$ 、碱炭比为 $1: 1$ 时, 可得极微孔 孔容为 $0.149 \mathrm{~mL} / \mathrm{g}$ 、极微孔率达 $36.3 \%$ 的微孔活性炭。活性炭的极微孔孔容与其在 $10^{4} \mathrm{~Pa}$ 时的 $\mathrm{CO}_{2}$ 吸附量高度线 性相关。

关 键 词: 活性炭; 极微孔; $\mathrm{KOH}$; 稻壳

中图分类号: TQ424 文献标识码: A

\section{Effects of Alkali/Char Ratio and Activation Temperature on Ultramicropores of Rice Husk Active Carbon}

\author{
LI Da-Wei ${ }^{1,2}$, MA Teng-Fei ${ }^{1,3}$, TIAN Yuan-Yu ${ }^{1,2}$, ZHU Xi-Feng $^{4}$, QIAO Ying-Yun ${ }^{2}$ \\ (1. College of Chemical Engineering, China University of Petroleum (East China), Qingdao 266580, China; 2. Research Centre \\ for Low-carbon Energy Sources, Shandong University of Science and Technology, Qingdao 266590, China; 3. PetroChina \\ Liaoyang Petrochemical Company, Liaoyang 111003, China; 4. Key Laboratory for Biomass Clean Energy of Anhui Province, \\ University of Science and Technology of China, Hefei 230026, China)
}

Abstract: Porous carbons were prepared by $\mathrm{KOH}$ activation of rice husk char at alkali/char ratios of $0.6: 1$ to $3: 1$ and activation temperatures from $640^{\circ} \mathrm{C}$ to $780{ }^{\circ} \mathrm{C}$. The obtained porous carbons were characterized using $\mathrm{N}_{2}$ adsorption and desorption technique, $\mathrm{CO}_{2}$ adsorption, TG-FTIR analyses. The results indicate that the ultramicropore size of the prepared porous carbons is mostly in the range of $0.42-0.70 \mathrm{~nm}$. As the alkali/char ratio increases, the volume of ultramicropores first rises and then diminishes, whereas the volume of ultramicropores tends to decrease monotonously with the increase of activation temperature. A microporous carbon with ultramicropore volume of $0.149 \mathrm{~mL} / \mathrm{g}$, ultramicropore fraction of $36.3 \%$, pore volume of $0.411 \mathrm{~mL} / \mathrm{g}$, and specific surface area of $774 \mathrm{~m}^{2} / \mathrm{g}$, can be produced by activation at $640^{\circ} \mathrm{C}$ at the alkali/char ratio of $1: 1$. The ultramicropore volume of the porous carbons and their $\mathrm{CO}_{2}$ uptake at $10^{4} \mathrm{~Pa}$ display a strong linear relationship.

Key words: active carbon; ultramicropore; $\mathrm{KOH}$; rice husk

收稿日期: 2014-05-13; 收到修改稿日期: 2014-07-13

基金项目: 国家自然科学基金(51206099); 中国科学院重点部署项目(KGZD-EW-304-3); 教育部新世纪优秀人才支持计划 (NCET-11-1031)

National Natural Science Foundation of China (51206099); Key Research Programof the Chinese Academy of Sciences (KGZD-EW-304-3); Program for New Century Excellent Talent in University of the Ministry of Education of China (NCET-11-1031)

作者简介: 李大伟(1982-), 男，讲师，博士后. E-mail: lidaweicumt@126.com

通讯作者：田原宇，教授.E-mail: tianyy1008@126.com 
活性炭具有比表面积高、孔结构发达、化学性 质稳定等优点，已广泛应用在吸附、分离、催化等 领域 ${ }^{[1-2]}$ 。活性炭的制备方法主要有物理活化法和化 学活化法。采用基于 $\mathrm{KOH}$ 的化学活化法, 尤其有利 于制备高比表面积的活性炭。迄今, 以 $\mathrm{KOH}$ 活化法 制备活性炭, 已有诸多研究 ${ }^{[3-6]}$ 。这些研究考察了制 备条件对比表面积、总孔容、微孔容积等孔结构参 数的影响, 但制备条件对极微孔影响的系统研究却 鲜见报道。

根据国际纯粹与应用化学会(IUPAC)对孔的分 类标准, 极微孔是指孔径小于 $0.7 \mathrm{~nm}$ 的孔道。研究 表明, 极微孔在活性炭吸附某些气体分子的过程中 发挥着重要作用。例如, De Souza 等 ${ }^{[7]}$ 发现, 低压时 活性炭的 $\mathrm{CO}_{2}$ 吸附量与其极微孔孔容密切相关; Silvestre 等 ${ }^{8}$ 发现，极微孔可以显著提高活性炭对 $\mathrm{H}_{2}$ 的吸附。可见, 研究极微孔在活化过程中的演变 规律, 对特定气体吸附剂的制备具有积极作用。

本工作考察了在 $\mathrm{KOH}$ 活化稻壳炭的过程中, 碱炭比和活化温度对活性炭极微孔的影响, 以期为 特定气体吸附剂的研制提供科学依据。

\section{1 实验方法}

\section{1 原料}

将稻壳水洗、干燥后储存备用, 其干基工业组成 为: 固定炭 $18.6 \mathrm{wt} \%$ ，挥发分 $70.4 \mathrm{wt} \%$, 灰分 $11.0 \mathrm{wt} \%$ 。 $\mathrm{KOH}(\mathrm{AR})$ 由国药集团化学试剂有限公司提供。实验 用水为自制去离子水。

\section{2 活性炭的制备}

将 $15 \mathrm{~g}$ 稻壳在 $400 \mathrm{~mL} / \mathrm{min}$ 的 $\mathrm{N}_{2}$ 下于 $520{ }^{\circ} \mathrm{C}$ 炭化 $20 \mathrm{~min}$, 所得稻壳炭记为 RHC。取粒径为 $0.15 \sim 0.90 \mathrm{~mm}$ 的 RHC $4.0 \mathrm{~g}$, 与溶于 $16 \mathrm{~mL}$ 水的 $\mathrm{KOH}$ 按 0.6: 1 3: 1 的碱炭比 $(\mathrm{KOH} / \mathrm{RHC}$, 按纯 $\mathrm{KOH}$ 计)混合, 密闭后 静置 $5 \mathrm{~h}$ 以上。之后, 将样品在 $\mathrm{N}_{2}(250 \mathrm{~mL} / \mathrm{min})$ 保 护下在 $140^{\circ} \mathrm{C}$ 干燥至恒重。所得样品置于镍舟, 再 移入管式炉, 通入 $400 \mathrm{~mL} / \mathrm{min}$ 的 $\mathrm{N}_{2}$ 。接着, 管式 炉以 $8^{\circ} \mathrm{C} / \mathrm{min}$ 的升温速率由室温升至 $400^{\circ} \mathrm{C}$ 并保温 $30 \mathrm{~min}$, 再以 $10^{\circ} \mathrm{C} / \mathrm{min}$ 升至 $640 \sim 780^{\circ} \mathrm{C}$, 保温 $60 \mathrm{~min}$ 。 最后, 将样品冷却至室温, 洗涤至中性, 并在 $110^{\circ} \mathrm{C}$ 干燥 $10 \mathrm{~h}$ 。

所得多孔炭记为 $\mathrm{K} n-m$, 其中 $\mathrm{K}$ 为 $\mathrm{KOH}, n$ 为碱 炭比, $m$ 为活化温度。例如, K1-710 代表以 $\mathrm{KOH}$ 为 活化剂, 采用 $1: 1$ 的碱炭比, 在 $710^{\circ} \mathrm{C}$ 活化制得的活 性炭。 $\mathrm{K} 1-0$ 为采用 $1: 1$ 的碱炭比, 在 $140^{\circ} \mathrm{C}$ 干燥至 恒重, 但不经后续加热而得到的含 $\mathrm{KOH}$ 稻壳炭。

\section{3 表征}

利用热重(Q500, TA Corp., USA)与红外(Nicolet 6700, Thermoscientific, USA) 联用仪，在线分析 RHC 和 K1-0 的热解过程及其气体产物。样品用量 约 $10 \mathrm{mg}$, 保护气 $\left(\mathrm{N}_{2}\right)$ 流量为 $70 \mathrm{~mL} / \mathrm{min}$, 升温速率 为 $10{ }^{\circ} \mathrm{C} / \mathrm{min}$, 光谱范围为 $4000 \sim 400 \mathrm{~cm}^{-1}$, 分辨率为 $4 \mathrm{~cm}^{-1}$ 。

利用比表面积及孔径分析仪(Tristar II 3020， 美国麦克), 测定样品在 $77 \mathrm{~K}$ 的 $\mathrm{N}_{2}$ 吸脱附等温线。 之后, 利用 BET 方程计算样品的比表面积 $\left(S_{\mathrm{BET}}\right)$, 利用 t-plot法计算样品的微孔孔容 $\left(V_{\text {micro }}\right)$, 利用 BJH 法确定中孔孔分布, 利用相对压力为 0.95 时所吸附 液氮体积计算总孔容 $\left(V_{\mathrm{t}}\right)$ 。中孔孔容 $\left(V_{\text {meso }}\right)$ 为 $V_{\mathrm{t}}$ 与 $V_{\text {micro }}$ 的差值, 微孔率 $\left(V_{\text {micro }} / V_{\mathrm{t}}\right)$ 为 $V_{\text {micro }}$ 与 $V_{\mathrm{t}}$ 的比值。

利用比表面积及孔径分析仪(ASAP 2020, 美国 麦克), 测定活性炭在 $0^{\circ} \mathrm{C}$ 下的 $\mathrm{CO}_{2}$ 吸附等温线。其 后, 利用 NLDFT 理论确定活性炭的极微孔孔容 $\left(V_{<0.7 \mathrm{~nm}}\right)$ 和极微孔孔分布。极微孔率 $\left(V_{<0.7 \mathrm{~nm}} / V_{\mathrm{t}}\right)$ 为 $V_{<0.7 \mathrm{~nm}}$ 与 $V_{\mathrm{t}}$ 的比值。

利用扫描电镜(S-4800, 日本日立公司)观察样 品的表面形貌。样品的干基灰分按国标 GB/T 12496.3-1999 进行测定。

\section{2 结果与讨论}

\section{1 多孔炭的表征}

图 1(a)为活性炭的 $\mathrm{N}_{2}$ 吸脱附等温线。这些等温 线在相对压力低于 0.1 时迅速上升, 而后几乎呈水 平线。由 IUPAC 的分类标准可知, 这些等温线属于 I 型, 说明样品的孔道以微孔为主。因此, 它们的微 孔率较高( $>70 \%$, 表 1), 但孔径 $>2 \mathrm{~nm}$ 的孔分布曲线 较低(图 1(b))。

由表 1 可知, 活性炭的比表面积均大于 $600 \mathrm{~m}^{2} / \mathrm{g}$, 远超过了 RHC $\left(114 \mathrm{~m}^{2} / \mathrm{g}\right)$, 表明 $\mathrm{KOH}$ 对稻壳炭产生 了显著的活化作用。此外，由表 1 可知，当碱炭比或 活化温度升高时, 微孔孔容显著增加, 致使总孔容 及比表面积显著增大, 这说明碱活化条件对活性炭 的孔结构产生了强烈影响。

由图 1(c)和(d)可知, 稻壳炭表面孔道极少, 而 活性炭表面则呈现丰富的微米级 $(0.5 \sim 6 \mu \mathrm{m})$ 管状大 孔, 在孔内壁上还显示出更小的孔道。可见, 稻壳炭 经活化之后, 孔道数量大幅增加, 这与活性炭的比 表面积远大于稻壳炭相一致。

\section{2 碱炭比对极微孔的影响}

图 2 为不同碱炭比下所得活性炭的极微孔孔分 

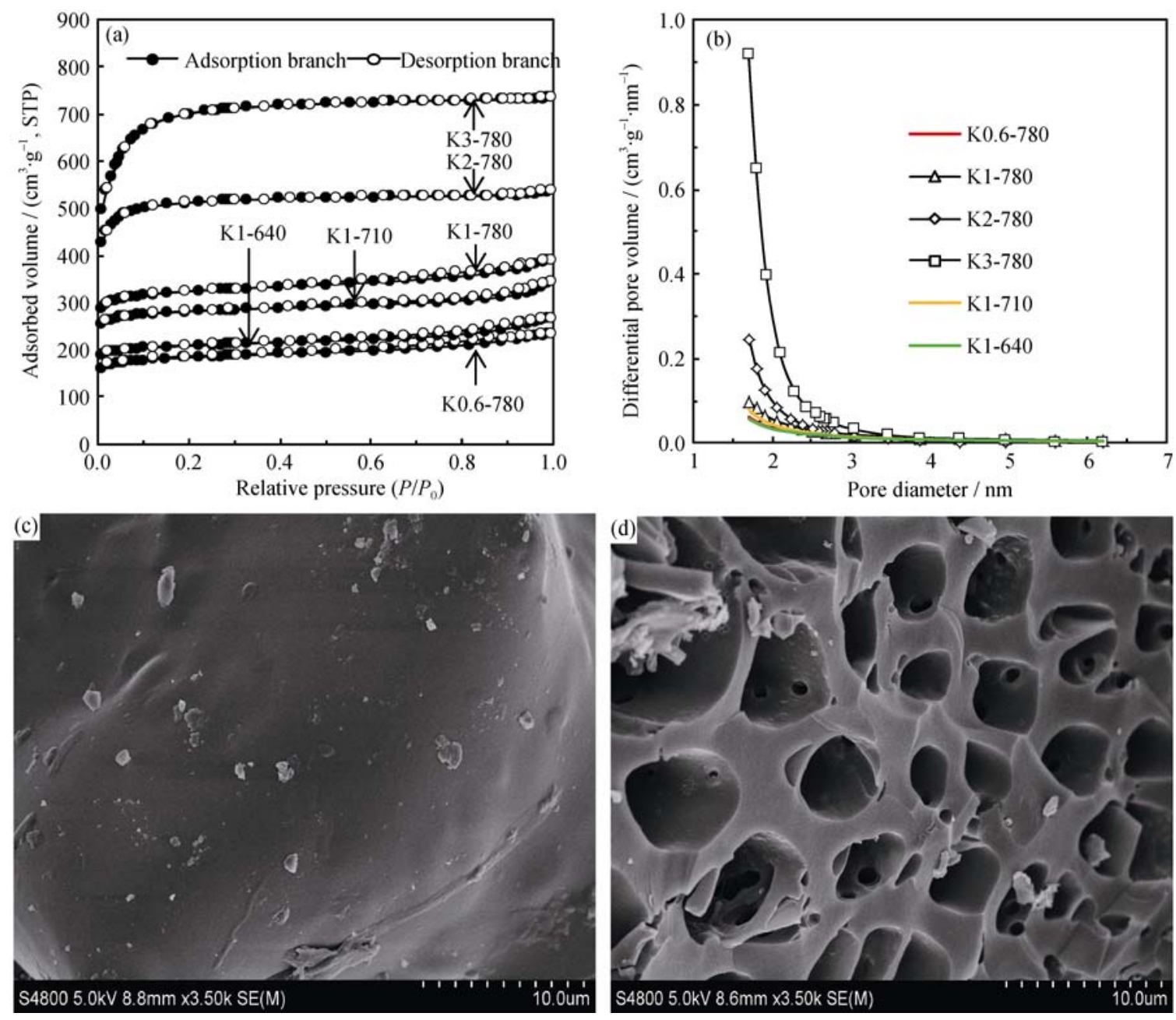

图 1 稻壳炭及活性炭的表征

Fig. 1 Characterizations for rice husk char and active carbons

(a) $\mathrm{N}_{2}$ adsorption/desorption isotherms; (b) Mesopore size distribution; (c) SEM image of rice husk char; (d) SEM image of K3-780

表 1 稻壳炭及活性炭的孔结构

Table 1 Textural properties of rice husk char and active carbons

\begin{tabular}{|c|c|c|c|c|c|c|c|c|c|}
\hline & Sample & $\begin{array}{c}S_{\mathrm{BET}} \\
/\left(\mathrm{m}^{2} \cdot \mathrm{g}^{-1}\right)\end{array}$ & $\begin{array}{c}V_{\mathrm{t}} / \\
\left(\mathrm{cm}^{3} \cdot \mathrm{g}^{-1}\right)\end{array}$ & $\begin{array}{c}V_{\text {micro }} \\
/\left(\mathrm{cm}^{3} \cdot \mathrm{g}^{-1}\right) \\
\end{array}$ & $\begin{array}{c}V_{\text {meso }} \\
/\left(\mathrm{cm}^{3} \cdot \mathrm{g}^{-1}\right)\end{array}$ & $\begin{array}{c}V_{<0.7 \mathrm{~nm}} \\
/\left(\mathrm{cm}^{3} \cdot \mathrm{g}^{-1}\right)\end{array}$ & $\begin{array}{c}V_{<1 \mathrm{~nm}} \\
/\left(\mathrm{cm}^{3} \cdot \mathrm{g}^{-1}\right)\end{array}$ & $\begin{array}{c}\left(V_{\text {micro }} / V_{\mathrm{t}}\right) \\
/ \% \\
\end{array}$ & $\begin{array}{c}\left(V_{<0.7 \mathrm{~nm}} / V_{\mathrm{t}}\right) \\
/ \%\end{array}$ \\
\hline \multirow{5}{*}{$\begin{array}{l}\text { Effects of } \\
\mathrm{KOH} / \text { char } \\
\text { ratio }\end{array}$} & RHC & 114 & 0.069 & 0.051 & 0.018 & - & - & - & - \\
\hline & K0.6-780 & 678 & 0.359 & 0.262 & 0.097 & 0.120 & 0.156 & 73.0 & 33.4 \\
\hline & K1-780 & 1199 & 0.600 & 0.479 & 0.121 & 0.139 & 0.196 & 79.8 & 23.1 \\
\hline & K2-780 & 1842 & 0.828 & 0.802 & 0.026 & 0.088 & 0.126 & 96.9 & 10.6 \\
\hline & K3-780 & 2695 & 1.137 & 1.106 & 0.031 & 0.078 & 0.117 & 97.3 & 6.9 \\
\hline \multirow{3}{*}{$\begin{array}{l}\text { Effects of } \\
\text { activation } \\
\text { temperature }\end{array}$} & K1-640 & 774 & 0.411 & 0.302 & 0.109 & 0.149 & 0.190 & 73.5 & 36.3 \\
\hline & K1-710 & 1041 & 0.527 & 0.422 & 0.105 & 0.149 & 0.202 & 80.1 & 28.3 \\
\hline & K1-780 & 1199 & 0.600 & 0.479 & 0.121 & 0.139 & 0.196 & 79.8 & 23.1 \\
\hline
\end{tabular}

布曲线。这些炭样的极微孔径均集中在 $0.42 \sim 0.70 \mathrm{~nm}$ 。 在 $0.42 \sim 0.51 \mathrm{~nm}$ 段, K1-780 的孔分布曲线与 K0.6-780 较为接近, 说明二者在该范围的孔容相当; 而在 $0.51 \sim 0.70 \mathrm{~nm}$ 段, K1-780 的孔分布曲线较高, 说明其在该范围的孔容较大。可见, 当碱炭比从 0.6 增大至 1 时, 0.51 0.70 $\mathrm{nm}$ 的孔得以发育, 而 $0.42 \sim$
$0.51 \mathrm{~nm}$ 的孔变化较小。当碱炭比继续增大时, 所得 活性炭(K3-780) 在 $0.42 \sim 0.70 \mathrm{~nm}$ 的孔分布曲线均低 于 K1-780, 表明该范围的孔容下降。这说明将碱炭 比从 1 增加至 3 , 不利于极微孔的发展。由图 3 还可 看出, K0.6-780、K1-780、K3-780 的最可几极微孔 孔径分别为 $0.56 、 0.59 、 0.57 \mathrm{~nm}$, 表明随着碱炭比 


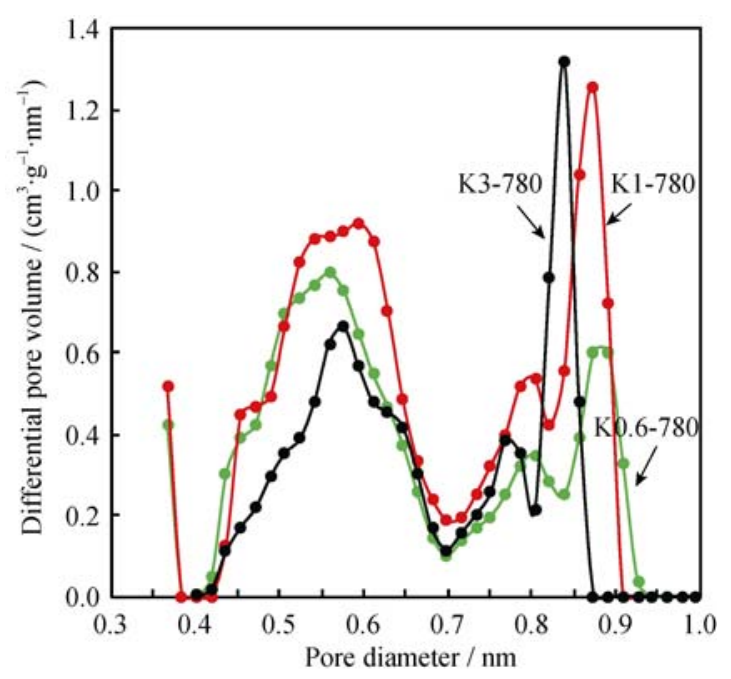

图 2 碱炭比对活性炭极微孔孔分布的影响

Fig. 2 Effects of alkali/char ratio on pore size distributions of ultramicropores of active carbons

的升高, 最可几极微孔孔径先增大后减小。这也反 映出, 将碱炭比从 0.6 增至 1 , 能促进极微孔的发育, 但继续提高碱炭比, 会减少极微孔。

表 1 列出了不同碱炭比下所得活性炭的极微孔 孔容, 由表 1 可知, 当碱炭比为 1 时, 极微孔孔容达 到最大值, 为 $0.139 \mathrm{~mL} / \mathrm{g}$ 。这是因为, 当碱炭比 $\leqslant 1$ 时, 增大碱炭比, 在高温下会有更多的活性物种与 稻壳炭活性位反应 ${ }^{[9]}$, 从而形成更多极微孔; 而当 碱炭比较高时 $(>1)$, 增大碱炭比会使孔壁变薄或孔 道连通 ${ }^{[9]}$, 致使极微孔扩展为较大的微孔。因此, 活 性炭 K3-780 的 $V_{\text {micro }}$ 大于 K1-780, 但其 $V_{<0.7 \mathrm{~nm}}$ 小于 K1-780(表 1)。

由表 1 还可知, 随着碱炭比的升高, 极微孔率 单调递减。这是因为, 当碱炭比增加时, 产生了大量 孔径较大的微孔 $(\geqslant 0.7 \mathrm{~nm})$, 微孔孔容 $\left(V_{\text {micro }}\right)$ 急剧 增加, 致使总孔容 $\left(V_{\mathrm{t}}\right)$ 的增幅显著超过了 $V_{<0.7 \mathrm{~nm}}$ 的 变化量。因此, $V_{<0.7 \mathrm{~nm}}$ 在 $V_{\mathrm{t}}$ 中所占比例, 随着碱炭比 的增加而持续降低。

\section{3 活化温度对极微孔的影响}

图 3 为不同活化温度下所得活性炭的极微孔孔 分布曲线, 由图可知, 这些活性炭的极微孔均集中 在 $0.42 \sim 0.70 \mathrm{~nm}$ 。在 0.42 0.57 nm 段, K1-640 的孔分 布曲线最高, 在该范围的极微孔孔容最大, 这说明低 活化温度利于较小极微孔的发育; 而在 $0.57 \sim 0.70 \mathrm{~nm}$ 段, K1-780 的孔分布曲线最高, 其极微孔孔容最大, 说明高活化温度可以促进较大极微孔的发育。这些 结果还说明, 随着活化温度的升高, 极微孔孔径呈 增大趋势。由图 3 还可得, K1-640、K1-710、K1-780 的最可几极微孔孔径分别为 $0.54 、 0.55 、 0.59 \mathrm{~nm}$, 说

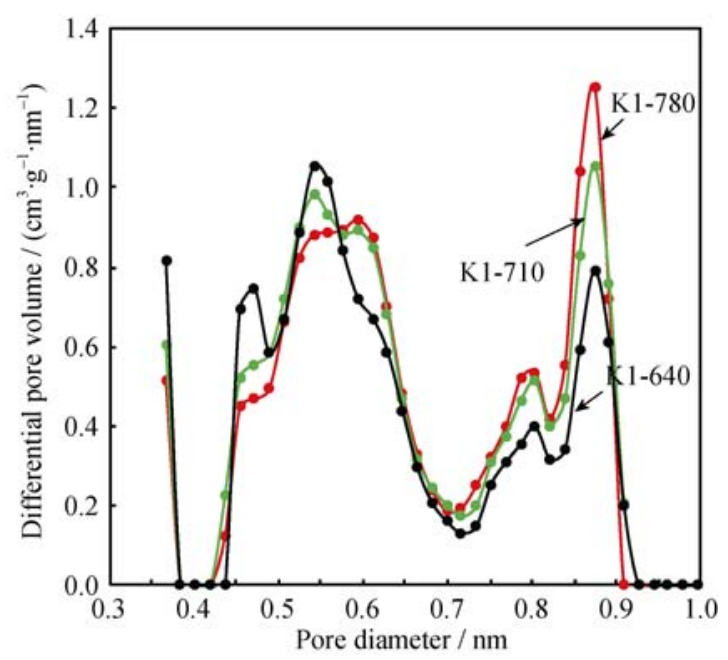

图 3 活化温度对活性炭极微孔孔分布的影响

Fig. 3 Effects of activation temperature on pore size distributions of ultramicropores of active carbons

明随着活化温度升高时, 最可几极微孔孔径增大, 这也说明极微孔孔径呈增大趋势。

不同活化温度下所得活性炭的极微孔孔容列于 表 1 。由表可知, 极微孔孔容最大值达到 $0.149 \mathrm{~mL} / \mathrm{g}$, 出现在活化温度较低时。随着活化温度的升高, 极微 孔孔容呈降低趋势。这是因为活化温度较高时, 活化 反应更为剧烈 ${ }^{[9]}$, 致使极微孔孔壁变薄甚至被烧穿, 从而扩展为大微孔甚至中孔。表 1 表明, K1-780 的 $V_{\text {micro }}$ 及 $V_{\text {meso }}$ 均大于 K1-640, 说明活化温度较高时, 微孔及中孔显著增多, 这与上述解释相吻合。

表 1 还表明, 活化温度升高时, 极微孔率单调 递减, 这是因为随着活化温度的升高, $V_{<0.7 \mathrm{~nm}}$ 因扩孔 效应呈减小趋势, 而 $V_{\mathrm{t}}$ 则因孔隙发育持续增大(表 1)。可见, 采用高活化温度不利于制备富含极微孔 的活性炭, 这与一些学者的研究结果相一致 ${ }^{[10]}$ 。

\section{4 孔结构演化机理}

稻壳炭的主要成分是 $\mathrm{SiO}_{2} \quad(\sim 32.4 \mathrm{wt} \%)$ 和 $\mathrm{C}$ $(54.5 \mathrm{wt} \%)$, 在与 $\mathrm{KOH}$ 接触并被加热的过程中, 可 以发生如下反应 ${ }^{[11-13]}$ :

$$
\begin{gathered}
\mathrm{SiO}_{2}+2 \mathrm{KOH} \rightarrow \mathrm{K}_{2} \mathrm{SiO}_{3}+\mathrm{H}_{2} \mathrm{O} \\
6 \mathrm{KOH}+2 \mathrm{C} \rightarrow 2 \mathrm{~K}+3 \mathrm{H}_{2}+2 \mathrm{~K}_{2} \mathrm{CO}_{3} \\
\mathrm{~K}_{2} \mathrm{CO}_{3} \rightarrow \mathrm{K}_{2} \mathrm{O}+\mathrm{CO}_{2} \\
\mathrm{~K}_{2} \mathrm{CO}_{3}+\mathrm{C} \rightarrow \mathrm{K}_{2} \mathrm{O}+2 \mathrm{CO} \\
\mathrm{K}_{2} \mathrm{O}+\mathrm{C} \rightarrow 2 \mathrm{~K}+\mathrm{CO} \\
2 \mathrm{~K}+\mathrm{CO}_{2} \rightarrow \mathrm{K}_{2} \mathrm{O}+\mathrm{CO}
\end{gathered}
$$

反应(1)在室温下即可发生。测试表明, 稻壳活 性炭的灰分(主要为 $\mathrm{SiO}_{2}$ ) 远低于稻壳炭 $(2.3 \sim 6.3 \mathrm{wt} \%<$ $32.4 \mathrm{wt} \%)$, 说明发生了反应(1), 致使 $\mathrm{SiO}_{2}$ 转化为水 溶性硅酸盐。

由图 4(b)可知, 在 $640^{\circ} \mathrm{C}$ 时, K1-0 位于 2217 

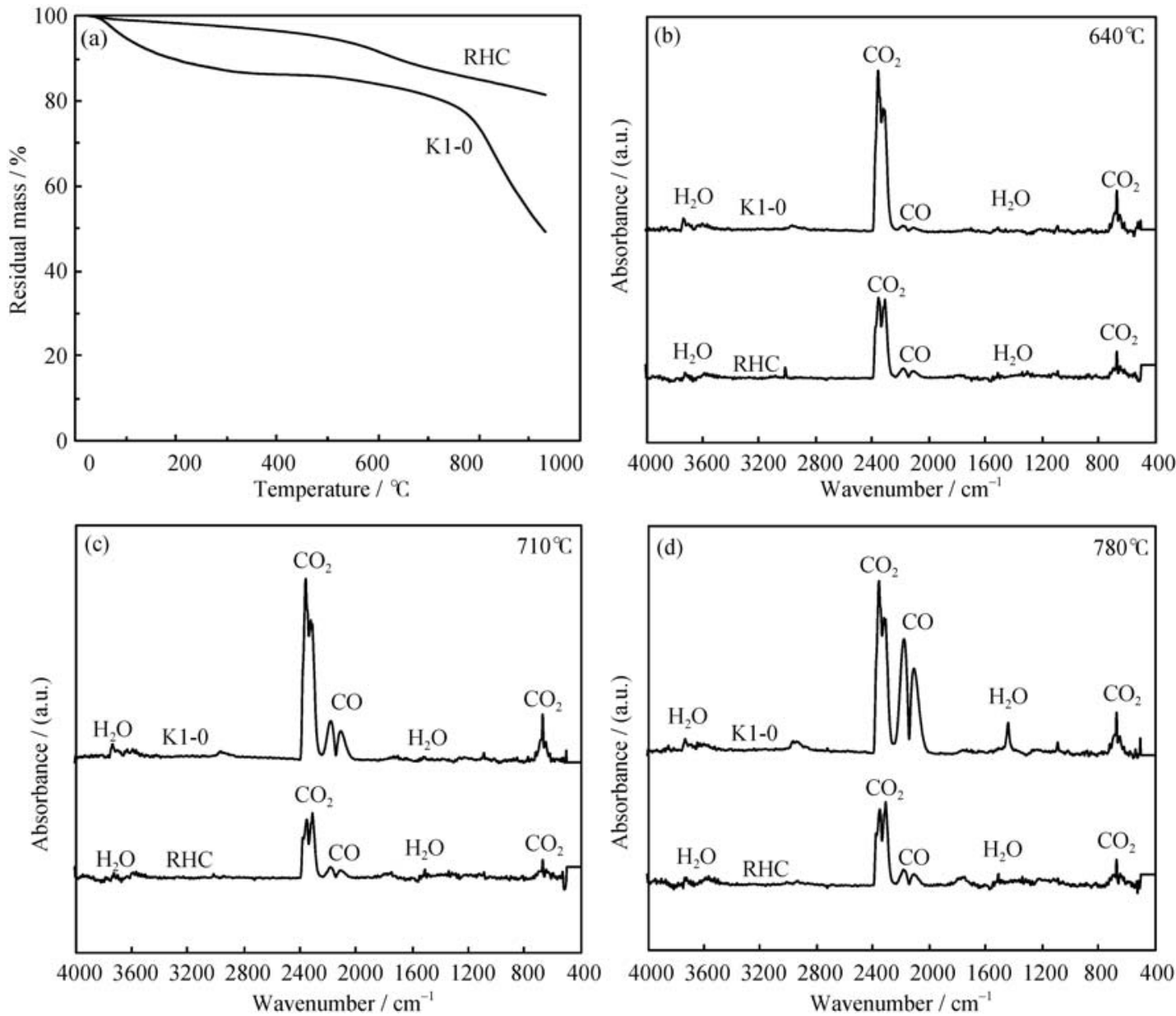

图 4 热解温度对稻壳炭(RHC)及含 $\mathrm{KOH}$ 稻壳炭(K1-0)重量及气体产物的影响

Fig. 4 Effects of heating temperature on mass and gaseous products of rice husk char (RHC) and $\mathrm{KOH}$-containing char (a) Thermogravimetric curves; FTIR spectra of gaseous products released at heating temperatures of $640^{\circ} \mathrm{C}(\mathrm{b}), 710^{\circ} \mathrm{C}(\mathrm{c})$ and $780^{\circ} \mathrm{C}(\mathrm{d})$

$2391 \mathrm{~cm}^{-1}$ 的 $\mathrm{CO}_{2}$ 特征峰 $^{[14]}$ 高于稻壳炭, 说明在稻 壳炭中加入 $\mathrm{KOH}$, 会促进 $\mathrm{CO}_{2}$ 的生成。这是因为反应 (2)约在 $400^{\circ} \mathrm{C}$ 发生 ${ }^{[15]}$, 生成的 $\mathrm{K}_{2} \mathrm{CO}_{3}$ 在 $477^{\circ} \mathrm{C}$ 即可与 炭反应生成 $\mathrm{CO}_{2}{ }^{[15]}$ 。需要说明的是, $\mathrm{K} 1-0$ 在 $640^{\circ} \mathrm{C}$ 时 释放的 $\mathrm{CO}_{2}$, 不应归因于反应(3), 因为反应(3)需 要在 $700^{\circ} \mathrm{C}$ 之上才能发生 ${ }^{[13]}$ 。另外, 由图 4(b)可知, 在 $640{ }^{\circ} \mathrm{C}$ 时, $\mathrm{K} 1-0$ 热解气的 $\mathrm{CO}$ 的特征峰 (2000 2250 $\left.\mathrm{cm}^{-1}\right)^{[14]}$ 不明显，说明在该温度下，不能 发生反应(4) (6), 因此它们不是 K1-640 出现极微孔 的原因。当温度从 $640^{\circ} \mathrm{C}$ 升高至 $780^{\circ} \mathrm{C}$ 时, $\mathrm{K} 1-0$ 的 $\mathrm{CO}$ 特征峰增大(图 4(b) (d)), 说明温度继续升高时 $\left(\geqslant 710{ }^{\circ} \mathrm{C}\right)$, 反应(4) (6)得以发生, 且呈加剧趋势, 使大量炭元素以 $\mathrm{CO}$ 形式脱离 K1-0, 并使 K1-0 的重 量迅速降低(图 4(a))。

综上可知，由于活化反应的发生，会有 $\mathrm{SiO}_{2}$ 及 炭元素脱离稻壳炭 ${ }^{[16]}$, 这将导致极微孔等孔道的形成 或扩展。此外, 当活化温度高于单质钾的沸点 $\left(762^{\circ} \mathrm{C}\right)$ 时, 生成的钾单质为气态, 可穿行在稻壳炭的类石
墨微晶层片之间，致使类石墨微晶层片被撑开或扭 曲, 这对活性炭的孔结构可以产生较大影响 ${ }^{[9,15]}$ 。

\section{5 极微孔对低压 $\mathrm{CO}_{2}$ 吸附量的影响}

图 5 为活性炭的极微孔孔容对其低压 $\mathrm{CO}_{2}$ 吸附 量 $\left(10^{4} \mathrm{~Pa}\right)$ 的影响。由图可知, 极微孔孔容越大, 低

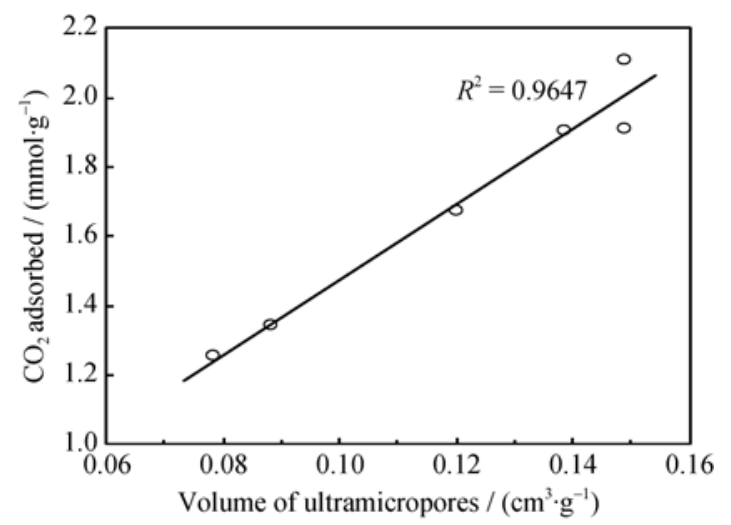

图 5 极微孔孔容与 $10^{4} \mathrm{~Pa}$ 下 $\mathrm{CO}_{2}$ 吸附量的关系

Fig. 5 Relation between ultramicropore volume and $\mathrm{CO}_{2}$ uptake at $10^{4} \mathrm{~Pa}$ 
压 $\mathrm{CO}_{2}$ 吸附量越大, 二者具有较强的相关性, 这与 $\mathrm{Hao}$ 等 ${ }^{[17]}$ 的报道相一致。拟合结果表明, 极微孔孔 容与低压 $\mathrm{CO}_{2}$ 吸附量的线性相关系数高达 0.9647 , 这再次表明二者密切相关。可见, 研究活化条件对 极微孔的影响, 对制备特定气体吸附用活性炭具有 积极意义。

\section{3 结论}

采用不同碱炭比 $(0.6: 1 \sim 3: 1)$ 和 活化温度 $\left(640 \sim 780{ }^{\circ} \mathrm{C}\right)$ 制备的稻壳活性炭, 其极微孔主要分布 在 $0.42 \sim 0.70 \mathrm{~nm}$, 最可几极微孔孔径为 $0.54 \sim 0.59 \mathrm{~nm}$ 。 当碱炭比增加时, 极微孔孔容先增大后减小; 而当 活化温度升高时, 极微孔孔容呈降低趋势。极微孔 率随碱炭比或活化温度的升高单调递减。在活化温 度为 $640^{\circ} \mathrm{C}$ 、碱炭比为 $1: 1$ 时, 可得极微孔孔容为 $0.149 \mathrm{~mL} / \mathrm{g}$ 、极微孔率为 $36.3 \%$ 、比表面积达 $774 \mathrm{~m}^{2} / \mathrm{g}$ 的微孔活性炭。多孔炭的极微孔孔容与其低压 $\mathrm{CO}_{2}$ 吸附量具有较高的线性相关性。

\section{参考文献:}

[1] XIE QIAN, ZHANG XIANG-LAN, LI LAN-TING, et al. Porosity adjustment of activated carbon: theory, approaches and practice. New Carbon Materials, 2005, 20(2): 183-190.

[2] TAN SAN-XIANG, TANG SHAO-ZAO, LIU YING-LIANG, et al. Preparation and antibacterial property of copper-loaded activated carbon microspheres. Journal of Inorganic Materials, 2010, 25(3): 299-306.

[3] PARASKEVA P, KALDERIS D, DIAMAdOPOUlOS E. Production of activated carbon from agricultural by-products. J. Chem. Technol. Biotechnol., 2008, 83: 581-592.

[4] DIAS J M, ALVIM-FERRAZ M C M, ALMEIDA M F, et al. Waste materials for activated carbon preparation and its use in aqueous-phase treatment: a review. J. Environ. Manage., 2007, 85(4): 833-846.

[5] KE YI-HU, YANG ER-TAO, LIU XIN, et al. Preparation of porous carbons from non-metallic fractions of waste printed circuit boards by chemical and physical activation. New Carbon Materials, 2013, 28(2): 108-115.
[6] LABUS K, GRYGLEWICZ S, MACHNIKOWSKI J. Granular $\mathrm{KOH}$-activated carbons from coal-based cokes and their $\mathrm{CO}_{2}$ adsorption capacity. Fuel, 2014, 118: 9-15.

[7] DE SOUZA L K C, WICKRAMARATNE N P, ELLO A S, et al. Enhancement of $\mathrm{CO}_{2}$ adsorption on phenolic resin-based mesoporous carbons by KOH activation. Carbon, 2013, 65: 334-340.

[8] SILVESTRE-ALBERO A M, WAHBY A, SILVESTRE-ALBERO $\mathrm{J}$, et al. Carbon molecular sieves prepared from polymeric precursors: porous structure and hydrogen adsorption properties. Ind. Eng. Chem. Res., 2009, 48(15): 7125-7131.

[9] WANG XIU-FANG, TIAN YONG, ZHANG HUI-PING. Preparation and activation mechanism of high specific surface area coal-based activated carbon. CIESC Journal, 2009, 60(3): 734737.

[10] YANG R, LIU G Q, LI M, et al. Preparation and $\mathrm{N}_{2}, \mathrm{CO}_{2}$ and $\mathrm{H}_{2}$ adsorption of super activated carbon derived from biomass source hemp (Cannabis sativa 1.) stem. Microporous Mesoporous Mater., 2012, 158: 108-116.

[11] JIMENEZ V, RAMIREZ-LUCAS A, DIAZ J A, et al. $\mathrm{CO}_{2}$ capture in different carbon materials. Environmental Science \& Technology, 2012, 46(13): 7407-7414.

[12] GUO YU-PENG, YANG SHAO-FENG. Preparation of active carbon with high specific surface area from rice husks. Chemical Journal of Chinese Universities, 2000, 21(3): 335-338.

[13] WANG J C, HEERWIG A, LOHE M R, et al. Fungi-based porous carbons for $\mathrm{CO}_{2}$ adsorption and separation. J. Mater. Chem., 2012, 22(28): 13911-13913.

[14] YANG JING-BIAO, CAI NING-SHENG. A TG-FTIR study on catalytic pyrolysis of coal. Journal of Fuel Chemistry and Technology, 2006, 34(6): 650-654.

[15] LI DONG-YAN, ZHOU HUA-LEI, TIAN YA-JUN, et al. High-specific-surface-area ativated carbon from anthracite. Rare Metal Materials and Engineering, 2007, 36(1): 583-586.

[16] LI DA-WEI, ZHU XI-FENG. Rice husk-based activated carbons with high mesoporosity prepared by a combination of $\mathrm{CO}_{2}$ activation and boiling in an alkaline solution. New Carbon Materials, 2013, 28(5): 363-368.

[17] HAO W, BJORKMAN E, LILLIESTRALE M, et al. Activated carbons prepared from hydrothermally carbonized waste biomass used as adsorbents for $\mathrm{CO}_{2}$. Appl. Energy, 2013, 112: 526-532. 